\title{
Prevalence of hypertension and peripheral arterial disease in adults in Léogâne, Haiti
}

\author{
Joel C Boggan', Schatzi H McCarthy², Jessica E Pritchard², Grant O’Brien², David K Walmer \\ 1 Division of General Internal Medicine, Department of Medicine, Duke University Health System, Durham, North Carolina, USA, ${ }^{2}$ Family Health \\ Ministries, Chapel Hill, North Carolina, USA \\ Keywords: global health \\ https://doi.org/10.29392/joghr.3.e2019031
}

\section{Journal of Global Health Reports}

Vol. 3, 2019

\section{Background}

The prevalence of hypertension under different treatment guidelines and peripheral arterial disease are unknown in many low and middle-income populations.

\begin{abstract}
Methods
A community sample of adults $\geqslant 18$ years in Léogâne, Haiti, had blood pressure and ankle-brachial index (ABI) measurements in the Summers of 2014 and 2015 in addition to surveys to ascertain knowledge related to awareness and consequences of hypertension. Descriptive statistics for prevalence of hypertension and peripheral artery disease were performed. Sensitivity analyses were performed for hypertension prevalence under the Eighth Joint National Committee (JNC) and 2017 guidelines.
\end{abstract}

\section{Results}

Overall, 1039 adults participated, with 43.6\% (453/1039) having hypertension, per JNC 7 guidelines. Under JNC 8, 13 fewer participants would be considered hypertensive (42.3\%, or 440/1039 participants). Defining stage I hypertension under 2017 guidelines would lead to an additional 231 participants meeting criteria (66.3\%, or 702/1059 participants) but only 10 needing additional pharmacotherapy. Of 409 participants with ABIs, 31 (7.6\%) had an $\mathrm{ABI} \leqslant 0.9,11(2.7 \%)$ had an $\mathrm{ABI}<0.8$, and none had an $\mathrm{ABI}<0.5$. On surveys, $98.9 \%$ of participants $(1,024 / 1,035)$ endorsed awareness of hypertension and $84.7 \%(856 / 1,027)$ that hypertension could lead to general health problems. Overall, $71.5 \%(449 / 628)$ cited hypertension as a risk factor for heart attacks and 96.0\% (603/628) for strokes. However, few participants were aware hypertension could be asymptomatic $(8.0 \%$, or $82 / 1,028)$.

\section{Conclusions}

Hypertension is highly prevalent in a community sample of Haitian adults. Adopting newer guidelines would substantially increase the proportion of adults meeting the definition without substantially increasing those requiring treatment. The population has relatively low rates of peripheral artery disease, further indicating chronic risk factor modification may limit cardiovascular morbidity in this low-income population.

Hypertension is a major risk factor for several diseases and the leading preventable cause of death worldwide. ${ }^{1,2}$ Recent studies have shown nearly three times as many individuals with hypertension live in low- and middle-income countries (LMICs) as in high-income countries, and that the prevalence of hypertension is increasing in LMICs while declining in high-income countries. ${ }^{2-4}$ Additionally, individual patient awareness of personal hypertension in LMICs is approximately half that of high-income populations. ${ }^{2}$

Peripheral artery disease (PAD) and other atherosclerotic endpoints are complications of long-standing hypertension. ${ }^{5}$ Unlike hypertension, however, the burden of PAD has been less well-characterized in LMICs, though increasing incidence is expected. ${ }^{6-8}$ In Haiti, where published rates of hypertension in the adult population vary from $29.5 \%$ to $47.1 \%$, there are no reports of PAD prevalence. ${ }^{9-12}$ As determining PAD prevalence in addition to hypertension prevalence may identify additional cardiovascular risk-lowering pharmacotherapeutic options, we sought to characterize both hypertension and PAD prevalence in a convenience sample of Haitian adults.

\section{METHODS}

\section{POPULATION}

Adults ages 18 years and older in Léogâne, Haiti, were sampled to determine the prevalence of hypertension in this community in summer 2014 and summer 2015. Léogâne is 
a city of 90,000 residents historically associated with sugarcane production located approximately 18 miles southwest of Port-au-Prince, the capital, and was the epicenter of the 2010 earthquake. The Haitian population is predominantly of West African descent and the Haitian diet has been estimated to include very high levels of sodium intake (up to 30-35 grams daily). ${ }^{13}$

\section{PROCEDURES}

During the summer of 2014 and 2015, potential study participants were approached for participation at various geographic locations across the community. These sites were chosen in partnership with health workers who lived within the community to ensure a broad geographic sample. Potential respondents were identified from the houses nearest each chosen location. After providing consent, participants answered a survey in Haitian Kreyol administered by local translators focusing on general health status and risk factors, including knowledge as well as a previous diagnosis of hypertension (English version, Appendix). Following the survey, researchers measured blood pressure readings using an automated cuff (OMRON Model HEM 907XL, Bannockburn, IL) with the patient seated after a minimum of five minutes, as well as height and weight. In summer 2014, participants also had ankle-brachial indices (ABIs) checked while supine after a minimum of five minutes using a Doppler ultrasound kit (Hokanson Model MD6VR Chart Recorder, Bellevue, WA) to assess for evidence of PAD. An exclusion criterion for study participation for 2015 was prior participation in 2014 .

\section{DEFINITIONS}

Body mass index (BMI) was calculated using weight in kilograms and height in meters, with overweight defined as BMI 25.0-29.9 and obese as BMI $\geqslant 30.0 .^{14,15}$ Hypertension was defined according to the Seventh Joint National Committee (JNC 7) guidelines as systolic blood pressure (BP) $\geqslant 140$ $\mathrm{mmHg}$ or diastolic BP $\geqslant 90 \mathrm{mmHg}$. ${ }^{16}$ As the Eighth JNC (JNC 8 ) guidelines were published in the run-up to this study and the 2017 guidelines from multiple societies were released during drafting of this manuscript, sensitivity analyses were also performed per these guidelines. Based on JNC 8 parameters, patients $>60$ years of age considered to be hypertensive with systolic blood pressure $\geqslant 150 \mathrm{mmHg}$, rather than 140 mmHg. ${ }^{17} 2017$ guideline prevalence of hypertension grade was estimated as patients with systolic blood pressure 130-139 $\mathrm{mmHg}$ and/or diastolic blood pressure 80-89 $\mathrm{mmHg}$ considered stage I hypertensive, with those with either a higher systolic or diastolic pressure considered stage II hypertensive. ${ }^{17,18}$ Reduced ABIs were defined as secondary to mild arterial disease if $0.8-0.9$, secondary to moderate arterial disease if $0.5-0.8$, and secondary to severe arterial disease if $<0.5 .19$

\section{DATA ANALYSIS}

Descriptive statistics were performed on survey and measurement data as noted above. All data were analyzed using Stata v. 9.2 (College Station, TX, USA).

\section{Table 1. Population characteristics}

\begin{tabular}{lc}
\hline Age (years): & $\begin{array}{c}\text { Number, } \% \\
(\mathrm{~N}=1039)\end{array}$ \\
Mean age (SD) & $41.3(17.7)$ \\
Median age (IQR) & $37(27-52)$ \\
$<30$ & $343(33.0)$ \\
$30-39$ & $222(21.4)$ \\
$40-49$ & $163(15.7)$ \\
$50-59$ & $127(12.2)$ \\
$60-69$ & $101(9.7)$ \\
$70-79$ & $43(4.1)$ \\
$\geq 80$ & $40(3.9)$ \\
Female (\%) & $672(64.7)$ \\
Median Body Mass Index (IQR)* & 22.8 \\
-Overweight (\%)* & $(20.0-26.7)$ \\
-Obese (\%)* & $218(22.7)$ \\
Used any tobacco (\%) & $190(19.8)$ \\
Previous blood pressure check at any & $57(5.5)$ \\
Previous blood pressure check in & $858(82.6)$ \\
Reported comorbidities (\%): & $706(67.9)$ \\
\hline Told had hypertension in past & $295(28.4)$ \\
\hline
\end{tabular}

SD - standard deviation, IQR - interquartile range

********ady mass index, overweight \%, and obese \% are calculated from the 958 participants for whom both height and weight were available.

\section{ETHICAL APPROVAL}

The study was approved by the Institutional Review Board (IRB) at Duke University (Protocol \# B0671) as well as the Misyon Sante Fanmi Ayisyen IRB in Haiti (Protocol \# 016_5_2013) to ensure cross-cultural ethical standards were met.

\section{RESULTS}

Demographic results are presented in Table 1. Overall, 1039 adults were sampled during the study period, $65 \%$ of whom were female. Of these, $83.1 \%$ (858/1033) had previously had his or her blood pressure checked during his or her lifetime, and $28.8 \%$ of the total population $(295 / 1025$, or $34.4 \%$ of those having previous blood pressure recordings) reported having at least one prior elevated blood pressure. Only $5.5 \%$ $(57 / 1034)$ of subjects currently smoked tobacco at any frequency. Of the 958 participants with both a height and weight recorded, 218 (22.7\%) were overweight and 190 (19.8\%) were obese, respectively.

On a single reading, 43.5\% (452/1039) participants had an elevated blood pressure of $>139$ systolic and/or $>89$ diastolic per JNC 7 guidelines or were taking an antihypertensive medication as verified by the name of the medication. Of these 452 participants, 58.2\% (269) were female and 9 
Table 2. Participants meeting hypertension criteria, by age group and guideline $(\mathrm{N}=1039)$

\begin{tabular}{lcccc}
\hline & & \multicolumn{2}{c}{2017 Guidelines } \\
Age (years) & JNC 7 & JNC 8 & Stage 1 & Stage 2 \\
Overall population (\%) & $453(43.6)$ & $440(42.3)$ & $231(22.2)$ & $452(43.5)$ \\
$<30$ & $77(22.4)$ & $77(22.4)$ & $81(23.6)$ & $77(22.4)$ \\
$30-39$ & $77(34.7)$ & $77(34.5)$ & $61(27.4)$ & $77(34.5)$ \\
$40-49$ & $76(46.6)^{*}$ & $75(46.0)$ & $48(29.4)^{*}$ & $75(46.0)$ \\
$50-59$ & $84(66.1)$ & $84(66.1)$ & $20(15.7)$ & $84(66.1)$ \\
$60-69$ & $74(73.3)$ & $67(66.3)$ & $16(15.8)$ & $74(73.3)$ \\
$70-79$ & $35(81.4)$ & $32(74.4)$ & $1(2.3)$ & $35(81.4)$ \\
$\geq 80$ & $30(75.0)$ & $28(70.0)$ & $4(10.0)$ & $30(75.0)$ \\
\hline
\end{tabular}

JNC - Joint National Committee

"Includes one patient age 45 years with diabetes and diastolic blood pressure meeting threshold for treatment for patients with diabetes under JNC 7 and 2017 guidelines.

Table 3. Prevalence of peripheral arterial disease, by age group and severity

\begin{tabular}{lccc}
\hline Age (years) & \multicolumn{2}{c}{ Ankle-brachial indices (N=409) } \\
Overall population (\%) & Severe $(<0.5)$ & Moderate $(0.5-0.79)$ & Mild $(0.8-0.9)$ \\
$<30$ & $0(0.0)$ & $11(2.7)$ & $20(4.9)$ \\
$30-39$ & $0(0.0)$ & $3(0.7)$ & $10(2.4)$ \\
$40-49$ & $0(0.0)$ & $1(0.2)$ & $1(0.2)$ \\
$50-59$ & $0(0.0)$ & $1(0.2)$ & $2(0.5)$ \\
$60-69$ & $0(0.0)$ & $1(0.2)$ & $1(0.2)$ \\
$70-79$ & $0(0.0)$ & $1(0.2)$ & $1(0.2)$ \\
$\geq 80$ & $0(0.0)$ & $0(0.0)$ & $1(0.2)$ \\
\hline
\end{tabular}

were subjects who had a blood pressure of $<139 / 89$ reporting current antihypertensive use. One further patient with diabetes had a diastolic blood pressure $>80 \mathrm{mmHg}$, which met treatment thresholds under JNC 7. Hypertension positively correlated with age, ranging from $22.4 \%$ between ages $18-29$ to $83.1 \%$ at $>79$ years (Table 2 ). Overall, prevalence of elevated blood pressure surpassed $50 \%$ in the $6^{\text {th }}$ decade of life. Under JNC 8 parameters, thirteen fewer participants would be considered hypertensive, for a total prevalence of $42.3 \%$ (440/1039). Defining stage I hypertension under the 2017 guidelines would lead to an additional 231 participants (22.2\% of the study population) meeting criteria for hypertension, for a population prevalence of $66.3 \%$ (702/ 1059).

ABIs were measured on 409 participants in order to assess arterial disease (Table 3). Overall, 31 of these 409 participants (7.6\%) had an $\mathrm{ABI} \leqslant 0.9$, while 11 (2.7\%) had an $\mathrm{ABI}<0.8$, and none had an $\mathrm{ABI}<0.5$. Of participants with a reduced $A B I, 9$ were $\geqslant 65$ years. Of the 31 with any reduction in ABI, only 14 (45.1\%) were hypertensive on the single blood pressure measurement. Of those with an $\mathrm{ABI}<0.8$, 8 of 11 (72.7\%) were hypertensive. An additional three patients $(0.7 \%)$ had $\mathrm{ABI}>1.4$.

Overall, $98.9 \%$ of participants $(1,024 / 1,035)$ endorsed some awareness of hypertension, and a similar high frequency $(99.6 \%$, or $1,026 / 1,030)$ stated they would be willing to take a medication for hypertension. A high frequency of participants also endorsed awareness that hypertension generally led to health problems $(84.7 \%$, or $856 / 1,027)$ and specific outcomes such as heart attacks $(71.5 \%$, or $449 / 628)$ and strokes (96.0\%, or 603/628). Alternatively, few participants were aware hypertension could be asymptomatic $(8.0 \%$, or $82 / 1,028)$.

\section{DISCUSSION}

Our results demonstrate that hypertension is highly prevalent in a young Haitian population with significant rates of being overweight or obese. The prevalence of hypertension increased across the lifespan in this population, affecting more than half of participants $>50$ years of age. However, relatively few hypertensive patients had evidence of concurrent $\mathrm{PAD}$, as determined by $\mathrm{ABI}$ measurement.

Similar to previous individual studies in Haiti, our estimates of hypertension showed a substantial proportion of patients with hypertension across all ages. Dickstein et al., whose study was based on patients presenting to clinics in and around Léogâne, found $41.9 \%$ of the rural population and $29.5 \%$ of the urban population $>40$ years old had hypertension. ${ }^{9}$ Our study, in which patients were sampled within the community at large, showed a prevalence of $62.8 \%$ for elevated blood pressures in persons $>40$ years and $43.5 \%$ for the overall sample. These findings correspond closely to a study performed by Jean-Baptiste et al. in Port-au-Prince, where hypertension was noted in $69.1 \%$ of males and $67.2 \%$ of females $\geqslant 40$ years. ${ }^{10}$ This is compared to the population- 
wide estimated rates of $25.5 \%$ for men and $26.7 \%$ for women $\geqslant 20$ years based on imputed values from the Jean-Baptiste study and results from Mexico, Jamaica, and Grenada. 2,10

Using the latest guideline definition for hypertension in this population would increase the prevalence of disease across all age groups and by $55 \%$ overall. This is similar to recent work from a global sample of adults from 80 countries (including 127 adults in Haiti), wherein adoption of the newest hypertension definition would relatively increase the imputed hypertensive population globally by $64 \% .20$ Unfortunately, this study did not publish country-level rates of hypertension under any definition. ${ }^{20}$ In our population, the increase in hypertension with a definition change would be most pronounced in the younger age groups, with the hypertensive population $<40$ years almost doubling. A much smaller population increase would require pharmacotherapy, however, as only an additional 10 patients $(0.9 \%$ of the population) would be recommended for treatment based on stage I hypertension and age $\geqslant 65$ years. For context, a recent US analysis noted a recommended increase in medication use of $1.9 \%$ under 2017 guidelines based on the nationallyrepresentative National Health and Nutrition Examination Survey. ${ }^{21}$

Our findings regarding PAD are consistent with Global Burden of Disease project results. That project estimated PAD in LMICs to increase from $1.2 \%$ in aged 25 -29 yearold males and $4.0 \%$ in similarly aged females to $21.5 \%$ and $18.7 \%$, respectively, in those $>95$ years old. ${ }^{7}$ These estimates were taken from only 12 studies in LMICs, however, and only one of the studies included another nation, such as Haiti, meeting the World Bank's criteria for a low-income country. ${ }^{22,23}$ This single study, performed partially in the Central African Republic, found 15.0\% overall prevalence of $\mathrm{PAD}$ in persons $\geqslant 65$ years of age, with higher rates $(17.2 \%)$ in women than men (11.9\%). ${ }^{23}$ The existence of only one study emphasizes the need to further investigate PAD in low-income countries. In our population, only 9 of 31 participants with $\mathrm{PAD}$ were $\geqslant 65$ years, implying many possible patients may be missed by studying PAD in only elder populations in LMICs. Additionally, identifying younger patients with evidence of PAD may improve opportunities for risk factor modification.

It is encouraging that high rates of participants endorsed knowledge of hypertension as a health condition and downstream health effects. While some of this reported awareness may be due to social desirability bias, rates in this community are likely higher than the aggregated LMIC rate of $37.9 \%$ awareness from a systematic review. ${ }^{2}$ One possible reason for increased hypertension awareness in this community may be an influx of healthcare resources in the aftermath of the 2010 earthquake, which increased the number and reach of health care personnel over a several-year period. In spite of these resources, however, only nine patients were currently taking an antihypertensive medication they could name.

One limitation of this study is the use of a convenience sample. While different geographic areas in the community were targeted, random sites chosen by geographic identifiers were not used for sampling. However, the density of development in the community allowed for extensive sampling over the course of the study period that likely limited variation based on geography alone. This sample was not able to be further adjusted based on national population data, as no formal census has been undertaken within Haiti since 2003. An additional limitation was use of a single cross-sectional blood pressure measurement for determination of blood pressure values; however, this methodology allowed us to sample patients not presenting to a clinic setting. Additionally, we followed standardized measurement protocols with rest periods before measurement to minimize the influence of immediate work or activity on blood pressure. Moreover, recent work indicates that repeated measurements during a single period only reduces the population estimated to have hypertension by $3.9 \% .^{20}$ Furthermore, given so few participants were taking blood pressure medications, we do not anticipate additional significant differences to have been present if measured repeatedly in a clinic setting.

Programmatic implications of this work include the need to target a wide age range for efforts related to hypertension measurement and treatment. To that end, we plan to use clinical partners in the area to screen and treat hypertensive patients chronically. Fortunately, few persons in the community routinely use tobacco and relatively few have PAD, which signals an opportunity to focus on treating patients for hypertension to reduce incidence of comorbid vascular events. In patients in whom symptomatic PAD is identified, low-risk pharmacotherapy could be initiated with aspirin consistent with known beneficial evidence without substantially adding to health resource limitations surrounding cost. ${ }^{24-27}$

\section{CONCLUSIONS}

In conclusion, we found a community sample of Haitians had rates of hypertension similar to previous populations studied within the country when using older guidelines. Adopting a hypertension definition consistent with the 2017 guidelines would lead to 55\% higher prevalence of hypertension in this population although the number recommended for pharmacotherapy would only increase by $0.9 \%$. Additionally, we found lower rates of peripheral artery disease than noted in the only other study measuring ABIs in a LMIC. These findings identify opportunities for chronic risk-factor modification and management to limit incident cardiovascular disease in a low-income setting.

\section{ACKNOWLEDGEMENTS}

The authors wish to thank the following personnel for assisting with survey administration: Garland Austin, Lydia Bradford, Elle Gault, Zachary Morrow, Tanya Thomas, Lillian Zerihun, and June Zhang.

\section{FUNDING}

This work was supported through the Duke Student Research Training Program through Duke University. 


\section{COMPETING INTERESTS}

The authors completed the Unified Competing Interest form at ww.icmje.org/coi_disclosure.pdf (available upon request from the corresponding author), and declare no conflicts of interest.
CORRESPONDENCE TO:

Joel C. Boggan, M.D., M.P.H.

Hospital Medicine Team (111M)

Durham VA Medical Center

508 Fulton St.

Durham, NC 27705 USA

Joel.boggan@dm.duke.edu 


\section{REFERENCES}

1. Lim SS, Vos T, Flaxman AD, et al. A comparative risk assessment of burden of disease and injury attributable to 67 risk factors and risk factor clusters in 21 regions, 1990-2010: a systematic analysis for the Global Burden of Disease Study 2010. Lancet. 2012;380(9859):2224-2260. doi:10.1016/s0140-6736(1 2)61766-8

2. Mills KT, Bundy JD, Kelly TN, et al. Global disparities of hypertension prevalence and control: A systematic analysis of population-based studies from 90 countries. Circulation. 2016;134(6):441-450. doi:1 0.1161/circulationaha.115.018912

3. Roth GA, Huffman MD, Moran AE, et al. Global and regional patterns in cardiovascular mortality from 1990 to 2013. Circulation. 2015;132(17):1667-1678. do i:10.1161/circulationaha.114.008720

4. Forouzanfar MH, Liu P, Roth GA, et al. Global burden of hypertension and systolic blood pressure of at least 110 to $115 \mathrm{~mm} \mathrm{Hg}, 1990-2015$. JAMA. 2017;317(2):165. doi:10.1001/jama.2016.19043

5. Gerhard-Herman MD, Gornik HL, Barrett C, Barshes NR, Corriere MA, Drachman DE. AHA/ACC guideline on the management of patients with lower extremity peripheral artery disease: A report of the American College of Cardiology/American Heart Association Task Force on Clinical Practice Guidelines. Circulation.

2016;2017;135:e726-79:726-779. doi:10.1161/CIR.000 $\underline{0000000000471}$

6. Fowkes FG, Forster RB, Levin CE, Naidoo NG, Roy A, Shu C. Prioritization of treatments for lower extremity peripheral artery disease in low- and middle-income countries. Int Angiol. 2017;36:203-215.

7. Fowkes FGR, Rudan D, Rudan I, et al. Comparison of global estimates of prevalence and risk factors for peripheral artery disease in 2000 and 2010: a systematic review and analysis. Lancet. 2013;382(9901):1329-1340. doi:10.1016/s0140-6736(1 3)61249-0

8. Criqui MH, Aboyans V. Epidemiology of peripheral artery disease. Circ Res. 2015;116(9):1509-1526. doi:1 $\underline{0.1161 / \text { circresaha. } 116.303849}$

9. Dickstein Y, Neuberger A, Golus M, Schwartz E. Epidemiologic profile of patients seen in primary care clinics in an urban and a rural setting in Haiti, 2010-11. Int Health. 2014;6(3):258-262. doi:10.1093/i nthealth/ihu033
10. Jean-Baptiste E, Larco P, Charles-Larco N, Vilgrain C, Simon D, Charles R. Glucose intolerance and other cardiovascular risk factors in Haiti. Prevalence of Diabetes and Hypertension in Haiti (PREDIAH). Diabetes Metab. 2006;32(5):443-451. doi:10.1016/s12 $\underline{62-3636(07) 70302-6}$

11. Niska RW, Sloand E. Ambulatory medical care in rural Haiti. J Health Care Poor Underserved. 2010;21(1):70-80. doi:10.1353/hpu.0.0256

12. Shipp ML. Awareness status and prevalence of hypertension in a group of urban Haitians: findings of a population-based survey. Ethn Dis.

2001;11:419-430.

13. Jean-Charles RR. Challenges in hypertension: the haiti experience. J Clin Hypertens. 2014;16(2):97-98. $\underline{\mathrm{d}}$ oi:10.1111/jch.12241

14. Garrow JS, Webster J. Quetelet's index (W/H2) as a measure of fatness. Int J Obes. 1985;9:147-153.

15. National Heart, Lung, and Blood Institute. Clinical guidelines on the identification, evaluation, and treatment of overweight and obesity in adults: the evidence report. National Institutes of Health. Published online September 1998:98-4083.

16. Chobanian AV, Bakris GL, Black HR, Cushman WC, Green LA, Izzo JL. The Seventh Report of the Joint National Committee on Prevention, Detection, Evaluation, and Treatment of High Blood Pressure: the JNC 7 report. JAMA. 2003;289(19):2560. doi:10.10 01/jama.289.19.2560

17. James PA, Oparil S, Carter BL, et al. 2014 evidence-based guideline for the management of high blood pressure in adults: report from the panel members appointed to the Eighth Joint National Committee (JNC 8). JAMA. 2014;311(5):507-520. doi:1 0.1001/jama.2013.284427

18. Whelton PK, Carey RM, Aronow WS, et al. 2017 ACC/AHA/AAPA/ABC/ACPM/AGS/APhA/ASH/ASPC/ NMA/PCNA Guideline for the Prevention, Detection, Evaluation, and Management of High Blood Pressure in Adults: A Report of the American College of Cardiology/American Heart Association Task Force on Clinical Practice Guidelines. J Am Coll Cardiol. 2018;71(19):e127-e248. doi:10.1016/j.jacc.2017.11.00 $\underline{6}$ 
19. Aboyans V, Criqui MH, Abraham P, et al. Measurement and interpretation of the anklebrachial index: a scientific statement from the American Heart Association. Circulation. 2012;126(24):2890-2909. doi:10.1161/cir.0b013e3182 $\underline{76 \mathrm{fbcb}}$

20. Beaney T, Schutte AE, Tomaszewski M, et al. May Measurement Month 2017: an analysis of blood pressure screening results worldwide. Lancet Glob Health. 2018;6(7):e736-e743. doi:10.1016/s2214-109 $\mathrm{x}(18) 30259-6$

21. Muntner P, Carey RM, Gidding S, et al. Potential US population impact of the 2017 ACC/AHA High Blood Pressure Guideline. Circulation. 2018;137(2):109-118. doi:10.1161/circulationaha.11 $\underline{7.032582}$

22. The World Bank Group. World Bank Country and Lending Groups 2018. Accessed October 14, 2018. htt ps://datahelpdesk.worldbank.org/knowledgebase/arti cles/906519-world-bank-country-and-lending-groups

23. Guerchet M, Aboyans V, Mbelesso P, et al. Epidemiology of peripheral artery disease in elder general population of two cities of Central Africa: Bangui and Brazzaville. Eur J Vasc Endovasc Surg. 2012;44(2):164-169. doi:10.1016/j.ejvs.2012.05.019
24. Belch J, MacCuish A, Campbell I, et al. The prevention of progression of arterial disease and diabetes (POPADAD) trial: factorial randomised placebo controlled trial of aspirin and antioxidants in patients with diabetes and asymptomatic peripheral arterial disease. BMJ. 2008;337(oct16 2):a1840-a1840. doi:10.1136/bmj.a1840

25. Fowkes FGR, Price JF, Stewart MC, Butcher I, Leng GC, Pell AC. Aspirin for prevention of cardiovascular events in a general population screened for a low ankle brachial index: a randomized controlled trial. JAMA. 2010;303(9):841-848. doi:10.1001/jama.2010.2 $\underline{21}$

26. Catalano M, Born G, Peto R. Prevention of serious vascular events by aspirin amongst patients with peripheral arterial disease: randomized, double-blind trial. J Intern Med. 2007;261(3):276-284. doi:10.1111/ j.1365-2796.2006.01763.x

27. Schmit K, Dolor RJ, Jones WS, et al. Comparative effectiveness review of antiplatelet agents in peripheral artery disease. JAHA. 2014;3(6):e001330. d oi:10.1161/jaha.113.001330 